\title{
Development of a Prediction Model to Estimate the Risk of Walking Limitations in Patients with Total Knee Arthroplasty
}

\author{
Yong-Hao Pua, Felicia Jie-Ting Seah, Ross Allan Clark, Cheryl Lian-Li Poon, \\ John Wei-Ming Tan, and Hwei-Chi Chong
}

\begin{abstract}
Objective. Early and accurate risk prediction of walking limitations after total knee arthroplasty (TKA) is important for clinical and economic reasons. However, to our knowledge, no studies have systematically integrated multiple predictors into a single, clinically practical model. Our study aimed to develop a prediction model to estimate the risk of post-TKA walking limitations.

Methods. We performed a prospective cohort study of 1096 patients who underwent elective, primary TKA between July 2013 and September 2014. Candidate predictors included patient demographics, surgical factors, and pre- and early (1-mo) post-TKA functional measures. The outcome of interest was self-reported walking limitations at 6 months of post-TKA. We used multivariable proportional odds regression with bootstrap internal validation to develop the model.

Results. In all, $12 \%$ of patients reported walking limitations (maximum walk time $\leq 15$ min) at 6 months postsurgery. The main predictors of increasing levels of walking limitations were preoperative walking limitations (overall $\mathrm{p}<0.001$ ), higher levels of body mass index [interquartile range (IQR)-OR 1.3, 95\% CI 1.2-1.5], lower values of 1-month post-TKA gait speed (IQR-OR 1.9, 95\% CI 1.3-2.6), the presence of contralateral knee pain (OR 1.9, 95\% CI 1.2-3.0), and the use of a quadstick preoperatively (OR 3.5,95\% CI 1.7-7.3). The prediction model had an optimism-corrected concordance index of 0.71 .

Conclusion. A small but sizable proportion of patients with TKA had persistent mobility limitations. Our prediction model may help to risk-stratify patients, and external validation is required before the model can be used in clinical practice. (First Release December 1 2015; J Rheumatol 2016;43:419-26; doi:10.3899/jrheum.150724)
\end{abstract}

Key Indexing Terms:

KNEE REPLACEMENT

PROGNOSIS MOBILITY LIMITATIONS

In patients with advanced stages of symptomatic knee osteoarthritis, although a total knee arthroplasty (TKA) can effectively relieve knee pain and restore function, about $24 \%$ to $30 \%$ of the patients who were assessed at around 2 years ${ }^{1,2}$ postsurgery had moderate to severe walking limitations. Accordingly, early and accurate identification of at-risk patients would better direct resources toward preventive care

From the Department of Physiotherapy, Singapore General Hospital, Singapore; Faculty of Health Sciences, Australian Catholic University, Victoria, Australia.

Funded by the Singapore General Hospital Centre Grant (Bioengineering Core) from the National Medical Research Council, Singapore (ref: NMRC/CG/016/2013).

Y.H. Pua, PhD, Department of Physiotherapy, Singapore General Hospital; F.J. Seah, MS, Department of Physiotherapy, Singapore General Hospital; R.A. Clark, PhD, Faculty of Health Sciences, Australian Catholic University; C.L. Poon, MS, Department of Physiotherapy, Singapore General Hospital; J.W. Tan, BSc, Department of Physiotherapy, Singapore General Hospital; H.C. Chong, BSc, Department of

Physiotherapy, Singapore General Hospital.

Address correspondence to Dr. Y.H. Pua, Department of Physiotherapy,

Singapore General Hospital, Outram Road, Singapore 169608.

E-mail:puayonghao@gmail.com

Accepted for publication September 25, 2015. for them. While previous cohort studies have improved our understanding of the risk factors for post-TKA functional

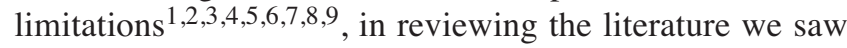
that no studies, to our knowledge, have systematically integrated multiple predictors into a single, clinically practical model. Thus, to facilitate clinical and shared decision making ${ }^{10}$, our study aimed to develop the first multivariable prediction model to estimate the risk of post-TKA walking limitations.

\section{MATERIALS AND METHODS}

Settings and participants. We conducted a prospective cohort study and identified 1352 patients aged 50 years or older who underwent a primary, unilateral TKA and postoperative outpatient physiotherapy at Singapore General Hospital, Singapore, from July 2013 to September 2014. We excluded patients who had a history of rheumatoid arthritis $(n=18)$, patients with stroke or Parkinson disease $(n=26)$, and patients who had revision TKA $(n=10)$. For patients with consecutive admissions for TKA $(n=57)$, only data from the first admission were analyzed to meet the statistical assumption of independence. Of the remaining 1241 patients who were evaluated preoperatively and were scheduled for 1-month and 6-month postoperative assessments, we selected a cohort of 1096 patients who had 6-month postoperative outcome data. All data were collected by physiother-

Personal non-commercial use only. The Journal of Rheumatology Copyright (c) 2016. All rights reserved. 
apists and data technicians and entered into an electronic registry database per routine practice policies of our institution. The institutional review board approved the study.

Candidate predictor variables. We considered candidate predictors a priori based on subject matter expertise and literature support for their association with self-report or performance-based physical function ${ }^{11,12}$. To improve the practicality of the prediction model, we considered variables that were less equipment-dependent and were routinely and easily measured in the clinical setting. For example, although patients with a recent TKA have joint effusion, limited knee range of motion, quadriceps weakness, and impaired balance control, we did not consider these variables because their quantification methods are often equipment-dependent and may vary across clinical settings. Instead, we chose to measure gait speed (described below), a simple but multidimensional measure that is related to the aforementioned knee impairments ${ }^{13,14}$. Finally, several variables were closely related - for example, the type of walking aids used preoperatively, at hospital discharge, and postoperatively - and we included 1 variable from each group of related variables in the model.

Altogether, we identified 11 predictors that included demographic and operative characteristics $1,2,3,4,6,8,9,15$, and measures of preoperative and early (1-mo) postoperative functional status ${ }^{2,3,4,5,7}$ (Table 1). Specifically, the presence of contralateral knee pain was measured by the "Patient Category" item (response choice B) from the Knee Society Clinical Rating System ${ }^{16}$. The presence of hypertension (HTN) was determined by patient self-report and it was chosen given its association with gait decline in older adults ${ }^{15}$. For falls history, the number of falls in the 1 year prior to TKA was collected through retrospective recall and we defined a fall as "an event which results in a person coming to rest inadvertently on the ground or other lower level and other than as a consequence of the following: sustaining a violent blow, loss of consciousness, sudden onset of paralysis as in a stroke, or an epileptic seizure" ${ }^{17}$. We classified patients into 1 of 3 groups: no fall, single fall (1 fall), and recurrent falls ( 2 or more falls). For the type of walking aids used preoperatively, we coded the responses into 4 categories: (1) none, (2) walking stick or umbrella, (3) quadstick, and (4) walking frame or 2-canes or crutches. For type of surgeons (specialist or general orthopedic surgeon), our patients were cared for by 42 orthopedic surgeons, 7 of whom were adult reconstruction specialists.

For the fast gait test, which was done 4 weeks post-TKA, patients stood directly behind the start line and were clocked from the time the first foot crossed the start line until the lead foot crossed the 10 -m finish line. Patients were instructed to "walk as quickly as you can, but safely" and to finish at least $2 \mathrm{~m}$ past the finish line to eliminate the deceleration effects from stopping the walk. Each patient performed a familiarization trial (at a comfortable pace) before the actual trial. Notably, although we measured habitual and fast gait speeds in our study, we selected fast gait speed because it has been shown to correlate closely with physical frailty ${ }^{18}$, functional decline $^{19}$, and onset of disability ${ }^{20}$ in older adults. Immediately after the fast gait test, participants were asked to rate their (ipsilateral) knee pain intensity using an 11-point numeric pain scale, with 0 indicating "no pain" and 10 indicating "worst pain ever experienced".

Outcome measure. The outcome of interest was the postoperative walking limitations. Notably, we chose an intermediate (6-mo) postoperative timepoint because it is a timepoint at which self-report physical function was reportedly nearing its peak ${ }^{21}$. In our study, patients were asked, both pre- and 6 months post-TKA, to estimate the time they were able to walk (without a rest) before they had severe difficulty with the operated knee. This variable had 4 categories: (1) > $30 \mathrm{~min}$, (2) 16 to $30 \mathrm{~min}$, (3) 5 to 15 min, and (4) around the house only.

Statistical analysis. Continuous variables were presented as means with SD and medians with interquartile range (IQR) while categorical variables were presented as percentages with frequencies. We used a transition plot to show the crossover between the 4 ordinal categories of walking limitations from the preoperative to 6 months postoperative timepoints.

To develop the prediction model, we used a proportional odds regression model which examined the multivariable association of the predictors listed in Table 1 with postoperative walking limitations. To optimize statistical power, we analyzed our outcome as a 4-level ordinal variable and quantified the model's accuracy in predicting the risk of walking limitations (defined

Table 1. Demographic and clinical characteristics of patients with and without walking limitations (defined as a maximum walk time $\leq 15$ min). Values appearing as "A, B, C" represent (A) the lower quartile, (B) the median, and (C) the upper quartile for continuous variables. Values appearing as D \pm E are mean \pm 1 SD

\begin{tabular}{|c|c|c|c|c|}
\hline Characteristics & Missing, \% (n) & Walking Limitations, $\mathrm{n}=135$ & No Walking Limitations, $\mathrm{n}=961$ & Overall, $\mathrm{n}=1096$ \\
\hline Age, yrs & 0 & $61.5,69.7,75.0(68.4 \pm 9.0)$ & $61.2,66.2,72.0(66.7 \pm 7.4)$ & $61.2,66.7,72.5(66.9 \pm 7.6)$ \\
\hline Women, \% (n) & 0 & 77 (104) & $75(719)$ & $75(823)$ \\
\hline Hypertension, \% (n) & 0 & $76(102)$ & $68(657)$ & $69(759)$ \\
\hline Falls history, \% (n) & $4.9(54)$ & & & \\
\hline None & & 87 (114) & $89(812)$ & $89(926)$ \\
\hline Preop walking aids, \% (n) & $0.9(10)$ & & & \\
\hline None & & $50(68)$ & $73(693)$ & $70(761)$ \\
\hline Stick & & $32(43)$ & $22(211)$ & \\
\hline QuadStick & & $11(15)$ & $3(27)$ & $4(42)$ \\
\hline Frame & & $7(9)$ & $2(20)$ & $3(29)$ \\
\hline Contralateral knee pain, \% (n) & $0.5(6)$ & $67(90)$ & $56(532)$ & $57(622)$ \\
\hline $5-15$ mins & & $50(67)$ & $40(389)$ & $42(456)$ \\
\hline Around house & & $28(38)$ & $8(73)$ & $10(111)$ \\
\hline Week 4 fast gait speed, m/s, \% (n) & $30(326)$ & $0.54,0.74,0.87(0.71 \pm 0.29)$ & $0.69,0.88,1.08(0.89 \pm 0.28)$ & $0.68,0.87,1.06(0.87 \pm 0.29)$ \\
\hline Week 4 knee pain, $\%$ (n) & $30(326)$ & $0.00,0.00,1.00(0.78 \pm 1.38)$ & $0.00,0.00,0.00(0.52 \pm 1.34)$ & $0.00,0.00,0.00(0.55 \pm 1.35)$ \\
\hline
\end{tabular}

BMI: body mass index; Preop: preoperative. 
as a maximum walk time $\leq 15 \mathrm{~min}$ ). Of interest, proportional odds regression (1) better preserves the information content of ordinal outcomes compared with binary logistic regression, (2) estimates common OR over all possible cutpoints of the outcome variable, and (3) allows exceedance probabilities to be estimated ${ }^{11}$. We used a full-model approach and included all predictors in the model ${ }^{11}$; however, variables were excluded when the sign of their regression coefficients was not considered biologically plausible (the "sign-OK" method ${ }^{22}$ ). We evaluated the relative explanatory value of the predictors by their partial Wald chi-square statistic minus the predictors' degrees of freedom ${ }^{11}$. To avoid assuming linearity, we modeled all continuous predictors as restricted cubic splines ${ }^{11,23}$, unless there was insufficient evidence against the linearity assumption $(\mathrm{p}>0.20)$. To reduce selection bias from missing predictor values, we performed multiple imputation using the aregImpute function from the rms package ${ }^{11,24,25}$, and the imputation models included the outcome, predictors, and auxiliary variables (for example, gait data from other postoperative timepoints). To account for overfitting of the prediction model, we shrank the OR in the final model using penalized maximum likelihood methods ${ }^{26}$. To account for the clustering of patients within surgeons, which may bias the CI toward being too narrow, we calculated Huber-White robust estimates of standard errors and $\mathrm{CI}^{27}$.

We assessed model performance in 2 ways. First, we measured model discrimination by the generalized concordance index $(\mathrm{C}$-index $)$, where a value of 1.0 represents perfect discrimination and 0.5 represents no discrimination ("coin flip") 11 . Because a prediction model is expected to perform better (optimistically) in the development sample than in new (but similar) samples, bootstrap internal validation with cluster sampling ${ }^{28}$ was performed to shrink the C-index for "optimism"11. Second, we assessed model calibration using a locally weighted scatterplot smoothing calibration plot. We tested and confirmed the validity of the proportional odds assumption graphically and we used the $\mathrm{rms}^{24}$, ggplot $2^{29}$, and $\mathrm{Gmisc}^{30} \mathrm{R}$ packages for all analyses and graphing (www.r-project.org).

\section{RESULTS}

Table 1 shows the demographics and pre- and postoperative characteristics of the patients. Women accounted for three-quarters of the sample [mean age $67 \mathrm{yrs}$ (SD 7.6)]; mean body mass index (BMI) for all patients was $27.5 \mathrm{~kg} / \mathrm{m}^{2}$ (SD 4.5). Among the predictor variables, Week 4 fast gait speed and knee pain (during the gait test) had the highest proportion of missing data $(29.7 \%)$ while other predictors had very few $(<5 \%)$ or no missing values (Table 1$)$. Preoperatively, half of the patients (52\%, 95\% CI 49-55) reported an inability to walk for more than 15 min (outcome levels 3 or 4); at 6 months postsurgery, around 1 of every 10 patients (12\%, 95\% CI 10-14\%; Figure 1). Details on the number of patients in each outcome category are given in Appendix 1.

Table 2 shows the results of the proportional odds model while Figure 2 shows the partial effects of the top 5 predictors on the probability of walking $\leq 15 \mathrm{~min}$ at 6 months post-TKA. Based on the Wald chi-square minus $d f$ statistics, preoperative walking limitations was the strongest predictor. Also, patients with higher BMI (IQR-OR 1.3, 95\% CI 1.2-1.5), slower Week 4 gait speed (IQR-OR $1.9,95 \%$ CI 1.3-2.6), and contralateral knee pain (OR 1.9, 95\% CI $1.2-3.0)$, and patients who walked with a quadstick preoperatively (quadstick vs none, OR $3.5,95 \%$ CI 1.7-7.3) were more likely to report greater postoperative walking limitations. Figure 3 is a nomogram that allows probabilities of postoperative walking limitations of individual patients to be estimated by drawing a straight line from the "sum of total points" axis to the "predicted risk" axes. The total number of points is obtained by summing the points of individual predictors. To facilitate results interpretation, we created a Web-based calculator (sgh-physio.shinyapps.io/walkPredict).

The C-index of the prediction model was 0.72 , and after correction for optimism it was 0.71 , indicating moderately good discrimination with minimal model optimism $(<2 \%)$. Figure 4 shows the calibration plot, with the light dotted and solid lines representing the calibration accuracy of the original and (optimism-corrected) bootstrap models, respectively. The 2 lines were relatively close to the dashed line of unity (which represents perfect agreement between the observed and predicted values), indicating moderately good calibration.

\section{DISCUSSION}

In a sample of 1096 patients with TKA, 12\% had walking limitations (maximum walk time $\leq 15 \mathrm{~min}$ ) at 6 months postsurgery. Although this estimate is lower than the $52 \%$ of the same cohort assessed preoperatively (Figure 1), the percentage of patients with persistent mobility limitations is worrisome, and early identification of these patients is thus of great clinical relevance.

In our study, we sought to develop a prediction model for post-TKA walking limitations and found that the strongest predictor was the preoperative level of walking limitations (Figure 2). This finding is unsurprising and consistent with data from several studies $2,3,4,5,7$, demonstrating that post-TKA functional limitations were strongly influenced by the same functional measure obtained preoperatively. Nonetheless, preoperative levels of walking limitations alone cannot fully predict the risk of postoperative walking limitations (Figure 1); hence, to further improve the predictive accuracy of the model, we included 2 complementary measures of functional ability - the preoperative use of a walking aid (Wald chi-square $=14.9, \mathrm{p}<0.01$ ) and 1 -month postoperative fast gait speed (Wald chi-square $=12.5, \mathrm{p}<$ $0.01)$, which added independent prognostic information (Figure 2 and Table 2).

Of interest, we found that the presence of contralateral knee pain was an independent predictor. This finding agrees with data from the Osteoarthritis Initiative study showing a statistically significant association between contralateral knee pain severity and Western Ontario and McMaster Universities Osteoarthritis Index (WOMAC) scores of the operated knee obtained at 6 months post-TKA ${ }^{1,6}$. Further, our finding is generally consistent with data from Hawker, et $a l^{5}$ demonstrating a negative association between the number of painful hips or knees and WOMAC functional scores obtained at least 6 months after surgery. Given that bilateral knee pain is prevalent in our sample (57\%; Table 1), our results support

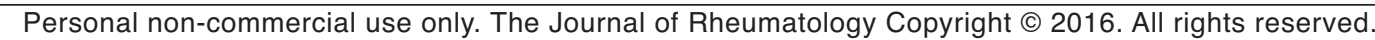




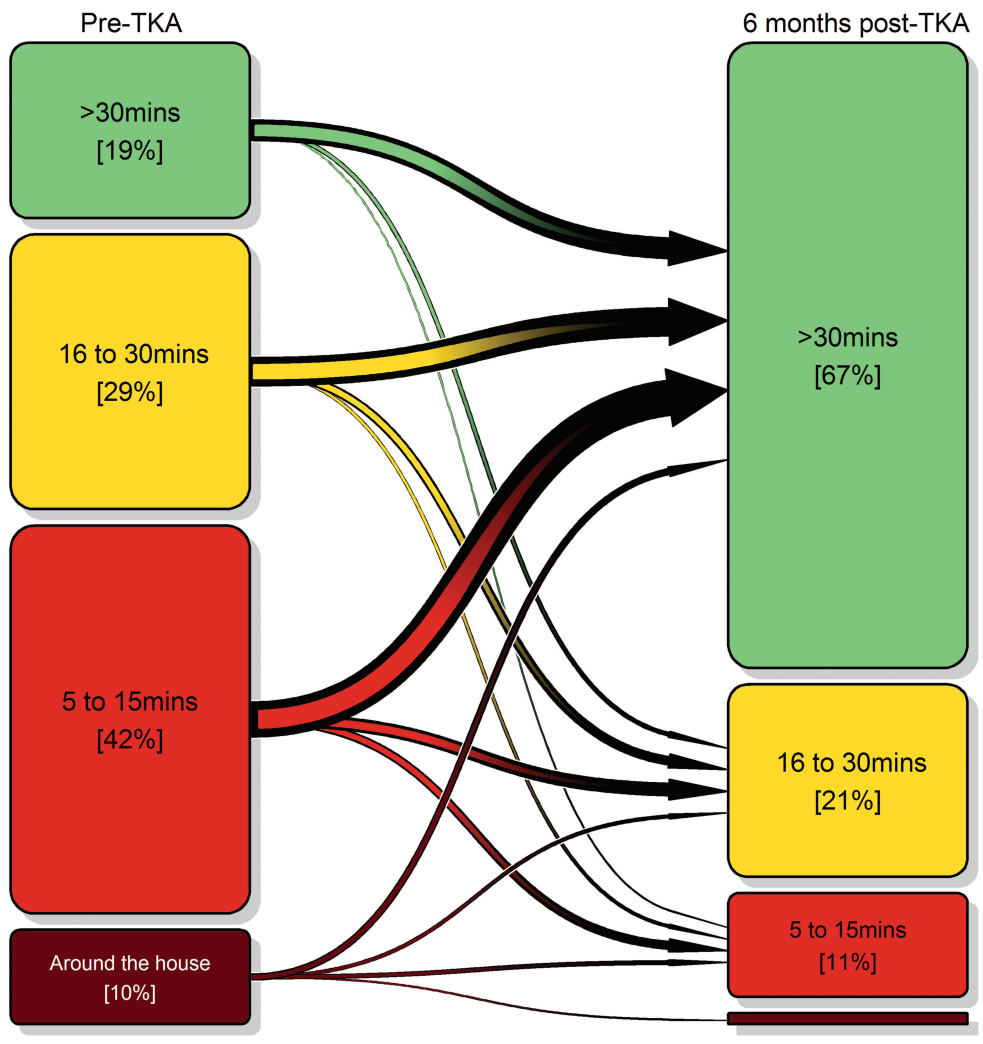

Figure 1. Crossover between 4 ordinal levels of self-report walking limitations in 1096 patients with TKA who were assessed preoperatively (left panel) and 6 months postoperatively (right panel). Box height and arrow size are proportional to the number of patients. TKA: total knee arthroplasty.
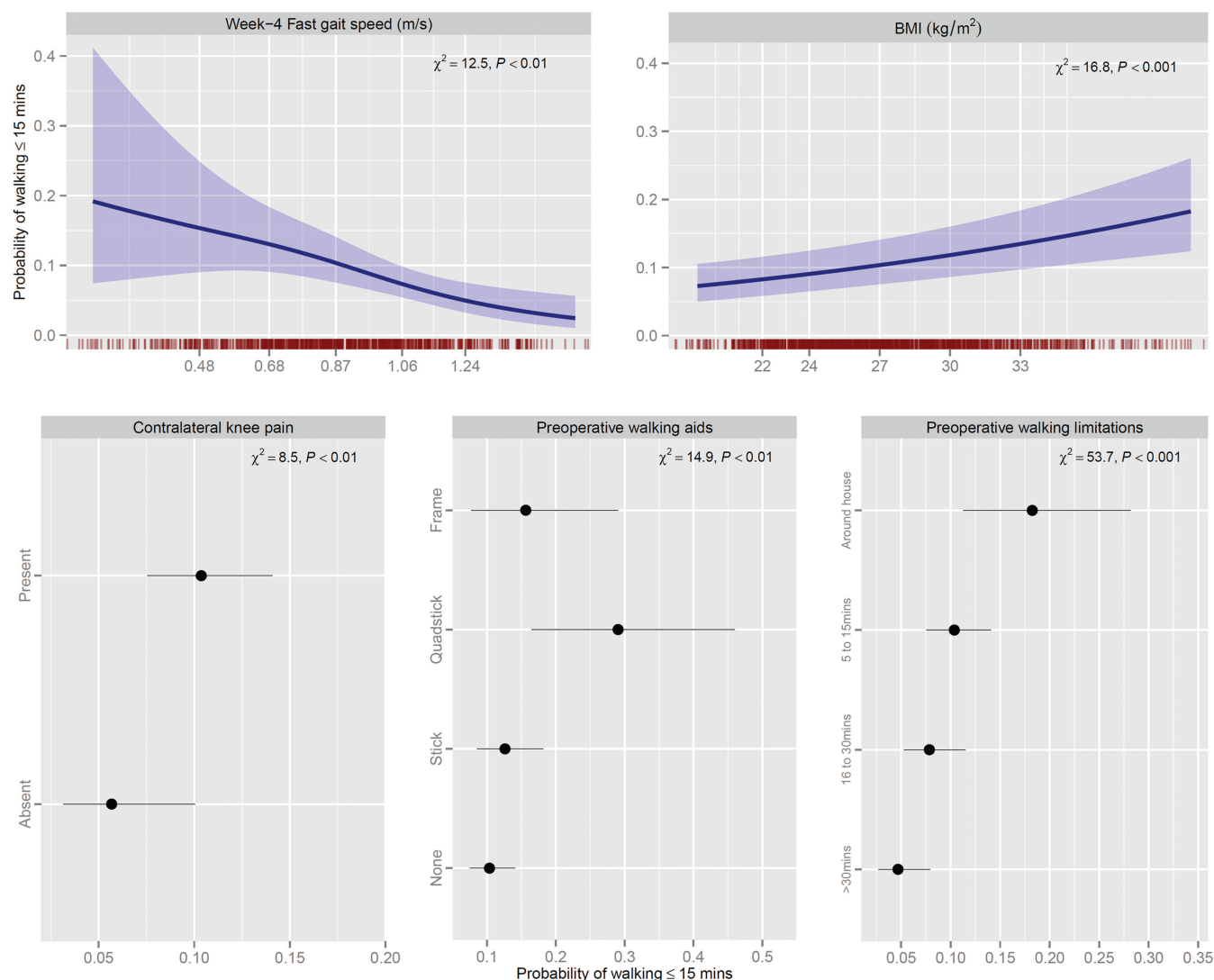

Figure 2. Partial effect of each of the top 5 predictors on the probability of walking $\leq 15$ mins at 6 months post-TKA after adjusting for other predictors. Predictors were ranked using the Wald chi-square in the proportional odds model. The $\mathrm{p}$ value is calculated using the Wald test. Shaded regions and error bars represent $95 \% \mathrm{CI}$ for the regression estimates. For fast gait speed and BMI, the observed values are indicated by the short vertical lines above the $\mathrm{X}$-axes. To further describe the spread of these continuous predictors, the tick labels indicate the 10th, 25th, 50th, 75th, and 90th percentile values. TKA: total knee arthroplasty; BMI: body mass index. 
Table 2. Multivariable association between predictors and walking limitations at 6 months postsurgery. OR (95\% CI) from proportional odds regression estimate the odds of greater walking limitations at the 75 th vs the 25th percentile for continuous predictors. For example, other variables being equal, a patient with a fast gait speed of $1.06 \mathrm{~m} / \mathrm{s}$ (75th percentile) had, on average, 0.54 times (95\% CI $0.38-0.77$ times) the odds of reporting greater walking limitations at 6 months post-TKA than did a patient who walked at $0.68 \mathrm{~m} / \mathrm{s}$ (25th percentile).

\begin{tabular}{|c|c|c|c|}
\hline Predictors & Comparison & OR $(95 \% \mathrm{CI})$ & $\mathrm{p}$ \\
\hline Age, yrs & 72.5 vs 61.2 & $1.07(0.85-1.34)^{*}$ & $<0.01$ \\
\hline BMI, $\mathrm{kg} / \mathrm{m}^{2}$ & 30.1 vs 24.3 & $1.34(1.16-1.54)$ & $<0.001$ \\
\hline Hypertension & Present vs absent (ref) & $1.41(0.98-2.03)$ & 0.06 \\
\hline \multirow{3}{*}{ Falls history } & & & 0.05 \\
\hline & Once vs none (ref) & $1.52(1.08-2.13)$ & \\
\hline & Recurrent vs none (ref) & $1.09(0.56-2.12)$ & \\
\hline \multirow[t]{4}{*}{ Preop walking aids } & & & $<0.01$ \\
\hline & Stick vs none (ref) & $1.25(0.96-1.62)$ & \\
\hline & QuadStick vs none & $3.54(1.72-7.27)$ & \\
\hline & Frame vs none & $1.60(0.82-3.14)$ & \\
\hline Contralateral knee pain & Present vs absent (ref) & $1.92(1.24-2.98)$ & $<0.01$ \\
\hline Adult reconstruction specialist & Yes vs no (ref) & $0.71(0.50-1.02)$ & 0.06 \\
\hline \multirow[t]{4}{*}{ Preop walking difficulty } & & & $<0.001$ \\
\hline & $>30$ mins vs around house (ref) & $0.22(0.13-0.36)$ & \\
\hline & 16-30 mins vs around house & $0.38(0.26-0.56)$ & \\
\hline & $5-15$ mins vs around house & $0.52(0.34-0.78)$ & \\
\hline Week 4 fast gait speed, $\mathrm{m} / \mathrm{s}$ & 1.06 vs 0.68 & $0.54(0.38-0.77)$ & $<0.01$ \\
\hline Week 4 knee pain, 10 -pt. scale & 5 vs 0 & $1.36(0.67-2.77)$ & 0.40 \\
\hline
\end{tabular}

\footnotetext{
* We modeled all continuous predictors using restricted cubic splines, and the $\mathrm{p}$ values are computed from their corresponding Wald tests for overall association. Although the $\mathrm{p}$ value may indicate statistical significance (and is correct), the comparison of the 75th and the 25th percentiles may yield a 95\% CI that includes 1.0. For knee pain intensity, because both 25 th and 75th percentiles were 0 , we compared pain scores of 5 and 0 instead. For categorical predictors, (ref) represents the reference group. For example, compared with a patient with no contralateral knee pain (the reference group), a patient with contralateral knee pain had, on average, 1.92 times (95\% CI 1.24-2.98 times) greater odds of reporting greater walking limitations.TKA: total knee arthroplasty; BMI: body mass index; Preop: preoperative; ref: reference.
}

Points for Individual Predictors
Age in years
BMI
Hypertension
Falls history
Preop walking aids
Contralateral knee pain
Adult recon. specialist
Preop walking ability
Wk-4 Fast gait speed ( $\mathrm{m} / \mathrm{s}$ )
Wk-4 Knee pain
Sum of Total Points
Predicted risk of walking $<=15 \mathrm{mins}$
Predicted risk of walking $<=30 \mathrm{mins}$

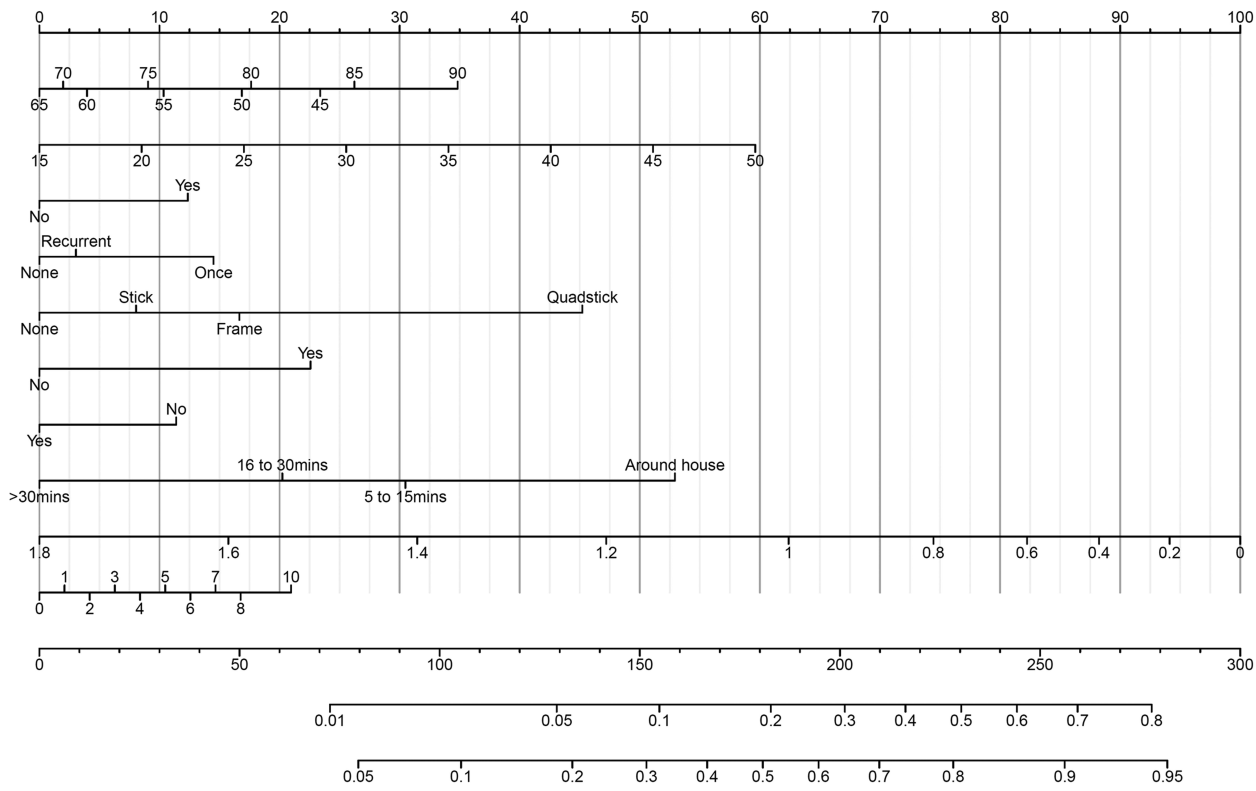

Figure 3. Nomogram for predicting risk of walking limitations 6 months post TKA. Instructions: Draw a vertical line from each predictor to the top line, labeled "Points for Individual Predictors", to calculate points associated with each predictor. The sum of these points is then marked on the line labeled "Sum of Total Points". Drop vertical lines from there to determine the predicted exceedance probabilities of walking $\leq 15$ mins or $\leq 30$ mins. TKA: total knee arthroplasty; BMI: body mass index; Wk: week. 


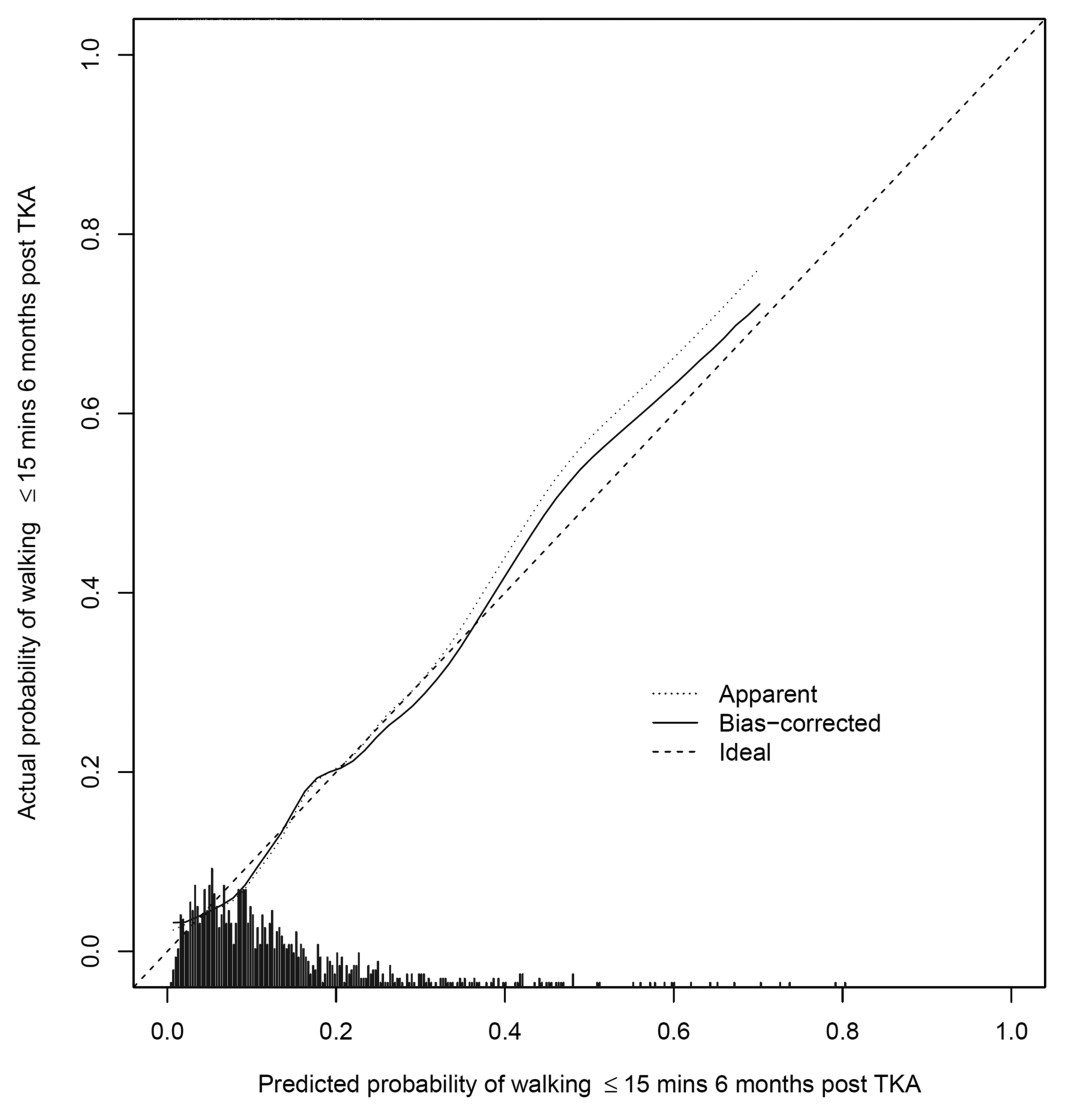

Figure 4. Calibration plot that illustrates the accuracy of the original prediction model ("Apparent") and the bootstrap model ("Bias-corrected") in predicting the probability of walking $\leq 15$ mins at 6 months post-TKA. Locally weighted scatterplot smoothing is used to model the relationship between observed and predicted probabilities. Spike histogram above the $\mathrm{X}$-axis shows the distribution of the predicted probabilities. TKA: total knee arthroplasty.

previous findings of a high prevalence of bilateral symptomatic knee OA in Asian Chinese ${ }^{31}$ patients. More importantly, the results suggest that to optimize functional outcomes, the contralateral knee should not be overlooked in the rehabilitation process.

It is also noteworthy that a high BMI was an influential predictor of greater post-TKA limitations in our model (Table 2 ) and these findings agree with those of several ${ }^{2,3,4,8,9}$, but not all ${ }^{5,7}$, previous studies. The reasons for these discrepant findings are unclear; however, a major strength of our study is that it encompassed a large sample of patients with a wide range of BMI values (16.6 to $46.6 \mathrm{~kg} / \mathrm{m}^{2}$ ). Further, we modeled BMI flexibly and avoided arbitrary BMI categorization $^{32}$. Thus, we believe that our results are robust, and if confirmed, they suggest that pre- and postoperative weight management may be indicated for at-risk patients with higher BMI.

Our study has limitations. First, our single-institution study raises uncertainty about the generalizability of our prediction model. However, our sample is nationally representative because our institution performed more than half
$(60 \%)$ of all knee arthroplasty in Singapore ${ }^{33}$. Nevertheless, we acknowledge the need to externally validate and update our model in new patient cohorts from our and (preferably) other institutions. Second, our study consisted of a preponderance of Asian women; hence, we cannot necessarily extend these results to non-Asian samples. Third, although many of our results support prior work, we acknowledge that more detailed predictors should be used. For example, future studies should consider contralateral knee pain on an ordinal rather than binary scale. Similarly, although we did not find the presence of self-report HTN to be an independent predictor (Table 2), we cannot conclude that more informative measures of HTN - such as systolic blood pressure measurements ${ }^{15}$ and the number of antihypertensive medications $^{34}-$ are not uniquely related to walking limitations. Fourth, our analysis focused on self-report walking duration and did not identify the full breadth of the walking limitation construct; hence, future studies should consider complementary, objective measures such as walking speed, walking endurance, and oxygen consumption during walking. Finally, for practical reasons, our predictors consisted primarily of 
self-report measures, and to the extent that performance-based measures such as muscle strength and postural balance may provide incremental predictive value, we acknowledge that the performance of our prediction model was adequate but certainly not optimal.

A small but sizeable proportion $(12 \%)$ of patients with TKA had walking limitations (maximum walk time $\leq 15 \mathrm{~min}$ ) at 6 months postsurgery. Our prediction model can potentially complement clinical and shared decision making by providing personalized risk estimates of future walking ability. External validation, however, is required before the model can be used in clinical practice.

\section{ACKNOWLEDGMENT}

We acknowledge the support from Bee-Yee Tan, the head of the Department of Physiotherapy, Singapore General Hospital. We also thank William Yeo from the Orthopaedic Diagnostic Centre, Singapore General Hospital, for his assistance. Finally, we thank Jasper Quek, Felicia Seet, Jennifer Suet-Ching Liaw, Jo Ann Sebarrotin, and Nai-Hong Chan for their assistance.

\section{REFERENCES}

1. Maxwell J, Niu J, Singh JA, Nevitt MC, Law LF, Felson D. The influence of the contralateral knee prior to knee arthroplasty on post-arthroplasty function: the multicenter osteoarthritis study. J Bone Joint Surg Am 2013;95:989-93.

2. Singh JA, O'Byrne M, Harmsen S, Lewallen D. Predictors of moderate-severe functional limitation after primary total knee arthroplasty (TKA): 4701 TKAs at 2-years and 2935 TKAs at 5-years. Osteoarthritis Cartilage 2010;18:515-21.

3. Dowsey MM, Nikpour M, Dieppe P, Choong PF. Associations between pre-operative radiographic changes and outcomes after total knee joint replacement for osteoarthritis. Osteoarthritis Cartilage 2012;20:1095-102.

4. Lingard EA, Katz JN, Wright EA, Sledge CB; Kinemax Outcomes Group. Predicting the outcome of total knee arthroplasty. J Bone Joint Surg Am 2004;86-A:2179-86.

5. Hawker GA, Badley EM, Borkhoff CM, Croxford R, Davis AM, Dunn $\mathrm{S}$, et al. Which patients are most likely to benefit from total joint arthroplasty? Arthritis Rheum 2013;65:1243-52.

6. Kahn TL, Soheili AC, Schwarzkopf R. Poor WOMAC scores in contralateral knee negatively impact tka outcomes: Data from the osteoarthritis initiative. J Arthroplasty 2014;29:1580-5.

7. Stevens-Lapsley JE, Petterson SC, Mizner RL, Snyder-Mackler L. Impact of body mass index on functional performance after total knee arthroplasty. J Arthroplasty 2010;25:1104-9.

8. Jones CA, Cox V, Jhangri GS, Suarez-Almazor ME. Delineating the impact of obesity and its relationship on recovery after total joint arthroplasties. Osteoarthritis Cartilage 2012;20:511-8.

9. Crosbie J, Naylor J, Harmer A, Russell T. Predictors of functional ambulation and patient perception following total knee replacement and short-term rehabilitation. Disabil Rehabil 2010;32:1088-98.

10. Barry MJ, Edgman-Levitan S. Shared decision making - pinnacle of patient-centered care. N Engl J Med 2012;366:780-1.

11. Harrell FE Jr. Regression modeling strategies: with applications to linear models, logistic regression, and survival analysis. New York: Springer; 2001.

12. Steyerberg E. Clinical prediction models: a practical approach to development, validation and updating. New York: Springer; 2009.

13. Pua YH. The time course of knee swelling post total knee arthroplasty and its associations with quadriceps strength and gait speed. J Arthroplasty 2015;30:1215-9.
14. Pua YH, Liang Z, Ong PH, Bryant AL, Lo NN, Clark RA. Associations of knee extensor strength and standing balance with physical function in knee osteoarthritis. Arthritis Care Res 2011;63:1706-14

15. Rosano C, Longstreth WT Jr, Boudreau R, Taylor CA, Du Y, Kuller $\mathrm{LH}$, et al. High blood pressure accelerates gait slowing in well-functioning older adults over 18-years of follow-up. J Am Geriatr Soc 2011;59:390-7.

16. Insall JN, Dorr LD, Scott RD, Scott WN. Rationale of the knee society clinical rating system. Clin Orthop Relat Res 1989:13-4

17. Zecevic AA, Salmoni AW, Speechley M, Vandervoort AA. Defining a fall and reasons for falling: comparisons among the views of seniors, health care providers, and the research literature. Gerontologist 2006;46:367-76.

18. Brown M, Sinacore DR, Binder EF, Kohrt WM. Physical and performance measures for the identification of mild to moderate frailty. J Gerontol A Biol Sci Med Sci 2000;55:M350-5.

19. Ko SU, Hausdorff JM, Ferrucci L. Age-associated differences in the gait pattern changes of older adults during fast-speed and fatigue conditions: results from the Baltimore longitudinal study of ageing. Age Ageing 2010;39:688-94.

20. Artaud F, Singh-Manoux A, Dugravot A, Tzourio C, Elbaz A. Decline in fast gait speed as a predictor of disability in older adults. J Am Geriatr Soc 2015;63:1129-36.

21. Kennedy DM, Stratford PW, Riddle DL, Hanna SE, Gollish JD. Assessing recovery and establishing prognosis following total knee arthroplasty. Phys Ther 2008;88:22-32.

22. Steyerberg EW, Eijkemans MJ, Harrell FE Jr, Habbema JD Prognostic modeling with logistic regression analysis: in search of a sensible strategy in small data sets. Med Decis Making 2001; 21:45-56.

23. Durrleman S, Simon R. Flexible regression models with cubic splines. Stat Med 1989;8:551-61.

24. Harrell FE Jr. rms: Regression modeling strategies. [Internet. Accessed October 21, 2015.] Available from: cran.R-project.Org/package $=$ rms

25. Moons KG, Donders RA, Stijnen T, Harrell FE Jr. Using the outcome for imputation of missing predictor values was preferred. J Clin Epidemiol 2006;59:1092-101.

26. Moons KG, Donders AR, Steyerberg EW, Harrell FE. Penalized maximum likelihood estimation to directly adjust diagnostic and prognostic prediction models for overoptimism: a clinical example. J Clin Epidemiol 2004;57:1262-70.

27. White H. A heteroskedasticity-consistent covariance matrix estimator and a direct test for heteroskedasticity. Econometrica 1980;48:817-38.

28. Bouwmeester W, Moons KG, Kappen TH, van Klei WA, Twisk JW, Eijkemans MJ, et al. Internal validation of risk models in clustered data: a comparison of bootstrap schemes. Am J Epidemiol 2013;177:1209-17.

29. Wickham H. ggplot2: elegant graphics for data analysis. New York: Springer; 2009.

30. Gordon M. Gmisc: descriptive statistics, transition plots, and more. [Internet. Accessed October 22, 2015.] Available from: cran.r-project.org/web/packages/Gmisc/index.html

31. Zhang Y, Xu L, Nevitt MC, Aliabadi P, Yu W, Qin M, et al. Comparison of the prevalence of knee osteoarthritis between the elderly Chinese population in Beijing and whites in the United States: the Beijing osteoarthritis study. Arthritis Rheum 2001;44:2065-71

32. Moons KG, Altman DG, Reitsma JB, Ioannidis JP, Macaskill P, Steyerberg EW, et al. Transparent reporting of a multivariable prediction model for individual prognosis or diagnosis (tripod): explanation and elaboration. Ann Intern Med 2015;162:W1-73.

Personal non-commercial use only. The Journal of Rheumatology Copyright (C) 2016. All rights reserved. 
33. Ministry of Health Singapore. Knee replacement surgery. [Internet. Accessed October 21, 2015.] Available from:

www.moh.gov.sg/content/moh_web/home/costs_and_financing/ HospitalBillSize/knee_replacementsurgery.html
34. Tinetti ME, Han L, Lee DS, McAvay GJ, Peduzzi P, Gross CP, et al. Antihypertensive medications and serious fall injuries in a nationally representative sample of older adults. JAMA Intern Med 2014;174:588-95.

APPENDIX 1. Number of patients in each outcome category at preoperative and postoperative levels.

\begin{tabular}{lcccc}
\hline Preoperative Level & & \multicolumn{2}{c}{ Postoperative Level } & \\
& $>30$ Mins & 16-30 Mins & 5-15 Mins & Around the House \\
\hline 30 mins & 169 & 30 & 6 & 1 \\
$16-30$ mins & 231 & 69 & 21 & 2 \\
$5-15$ mins & 286 & 103 & 65 & 2 \\
Around the house & 49 & 24 & 30 & 8 \\
\hline
\end{tabular}

